\title{
Localization of Brachytherapy Seeds in Ultrasound by Registration to Fluoroscopy
}

\author{
P. Fallavollita ${ }^{* 1}$, Z. KarimAghaloo ${ }^{2}$, E.C. Burdette ${ }^{3}$, D. Y. Song ${ }^{4}$, \\ P. Abolmaesumi ${ }^{5}$, and G. Fichtinger ${ }^{1}$ \\ ${ }^{1}$ Queen's University, Canada, \\ ${ }^{2}$ McGill University, Canada, \\ ${ }^{3}$ Acoustic MedSystems Inc, Illinois, \\ ${ }^{4}$ John Hopkins Hospital, Baltimore, \\ ${ }^{5}$ University of British Columbia, Canada
}

\begin{abstract}
Motivation: In prostate brachytherapy, transrectal ultrasound (TRUS) is used to visualize the anatomy, while implanted seeds can be seen in C-arm fluoroscopy or CT. Intra-operative dosimetry optimization requires localization of the implants in TRUS relative to the anatomy. This could be achieved by registration of TRUS images and the implants reconstructed from fluoroscopy or CT. Methods: TRUS images are filtered, compounded, and registered on the reconstructed implants by using an intensity-based metric based on a 3D point-to-volume registration scheme. A phantom was implanted with 48 seeds, imaged with TRUS and CT/X-ray. Ground-truth registration was established between the two. Seeds were reconstructed from CT/X-ray. Seven TRUS filtering techniques and two image similarity metrics were analyzed as well. Results: For point-to-volume registration, noise reduction combined with beam profile filter and mean squares metrics yielded the best result: an average of $0.38 \pm 0.19 \mathrm{~mm}$ seed localization error relative to the ground-truth. In human patient data $\mathrm{C}$-arm fluoroscopy images showed 81 radioactive seeds implanted inside the prostate. A qualitative analysis showed clinically correct agreement between the seeds visible in TRUS and reconstructed from intra-operative fluoroscopy imaging. The measured registration error compared to the manually selected seed locations by the clinician was $2.86 \pm 1.26 \mathrm{~mm}$. Conclusion: Fully automated seed localization in TRUS performed excellently on ground-truth phantom, adequate in clinical data and was time efficient having an average runtime of 90 seconds.
\end{abstract}

Keywords: Prostate brachytherapy, Registration, Fluoroscopy, Ultrasound

\section{INTRODUCTION}

Prostate cancer continues to be the second most common cancer in North American men, with an estimated 230,000 new cases per year ${ }^{1}$. Brachytherapy has emerged as one of the principal treatment options of early stage prostate cancer, for the majority of cases we diagnose nowadays. The procedure entails permanent implantation of small radioactive pellets, or seeds, the size of a rice grain into the prostate in order to eradicate the cancer with ionizing radiation while sparing surrounding healthy tissues. Faulty needle and seed placement often cause an insufficient dose to the cancer and/or inadvertent radiation of the rectum, urethra or bladder. The former causes failure of treatment while the latter results in adverse side effects like rectal ulceration, incontinence and painful urination. The ability to perform dosimetry optimization during the procedure could change the standard of care in brachytherapy, but such function is not available today and it is most unfortunate that implants are currently performed without an explicit dosimetry evaluation in the operating room ${ }^{2}$. Generally, dosimetric analysis requires precise localization of the implanted seeds in relation to the prostate and surrounding anatomy.

*pascal@cs.queensu.ca; phone: (613) 533-6000 Ext. 78234; Percutaneous Surgery Lab (Perk Lab), School of Computing, Queen's University, Kingston, Ontario, Canada, K7L 3N6. Z. Karim-Aghaloo and P. Abolmaesumi were with Queen's University during this research.

Medical Imaging 2010: Visualization, Image-Guided Procedures, and Modeling, edited by Kenneth H. Wong, Michael I. Miga, Proc. of SPIE Vol. 7625, 762519 - @ 2010 SPIE · CCC code: 1605-7422/10/\$18 · doi: 10.1117/12.844015 
Brachytherapy is predominantly performed with transrectal ultrasound (TRUS) guidance that provides adequate realtime visualization of the prostate but not of the implanted seeds. Despite significant efforts, localization of seeds directly from TRUS has not been clinically practical or robust. C-arm fluoroscopy is widely used for gross visual assessment of the implant, but it cannot show the prostate.

The published history of C-arm fluoroscopy in brachytherapy originates ${ }^{3}$ when it was first used as a solo guidance modality. Shortly after TRUS emerged as a primary image guidance modality, fluoroscopy became a secondary tool for gross visual observation. Mobile C-arms are ubiquitous in contemporary prostate brachytherapy, with over $60 \%$ of the practitioners using it for qualitative implant analysis during the procedure 4 in a setup shown in Figure 1-left. Recently, accurate reconstruction of seeds from fluoroscopy has become possible 5-7, but since it cannot show the prostate and soft tissues (Figure 1-right), fluoroscopy alone cannot be used for dosimetry. At a few specialized centers, CT imaging is available during brachytherapy for implant reconstruction. ${ }^{8}$ In all, as seeds can be reconstructed from fluoroscopy or CT, the remaining problem is registration of TRUS with the reconstructed seeds.
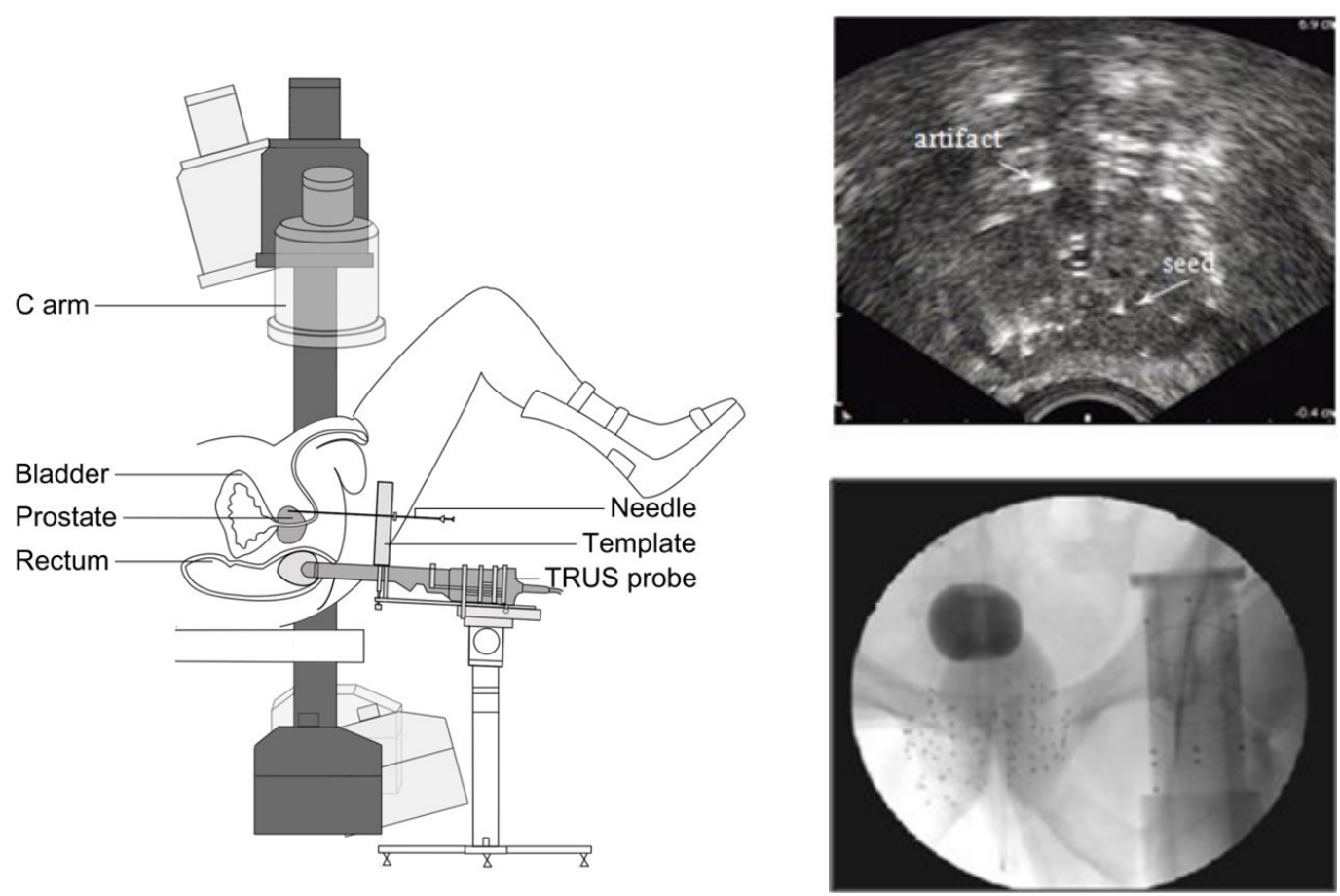

Figure 1. Prostate Brachytherapy Setup. (Left) Typical prostate brachytherapy setup with TRUS and C-arm fluoroscopy imaging. Note the severely limited rotation angle for the C-arm, of about 20 degrees about the AP axis. (Right) Transrectal ultrasound shows a faint trace of the prostate contour, some of the implanted seeds and various imaging artifacts. C-arm fluoroscopy images are acquired during the intervention to qualitatively validate seed positions. The prostate is not visible in fluoroscopy. Illustration by Xiao Xiao Ma.

To this end, Zhang et al. ${ }^{9}$ suggested affixing X-ray fiducials onto the TRUS probe. French et al. ${ }^{10}$ suggested using the TRUS probe as a registration fiducial. Jain et al. ${ }^{6}$ proposed mounting a fiducial structure above the abdomen, spatially calibrated with the template. In all the above, the registration took place through static fiducials and cannot account for motion of the anatomy between the TRUS and fluoroscopy sessions. When the TRUS probe is retracted from the rectum for fluoroscopy imaging, the prostate relaxes posteriorly. To counteract this problem, Su et al. suggested using a point-based registration between seeds directly reconstructed between TRUS and fluoroscopy. ${ }^{6,11}$ This requires exact localization of the seeds in TRUS, which as mentioned earlier, has not been possible with clinically sufficient accuracy and robustness. 
In this paper, we present a novel point set-to-volume registration procedure of intensity-based registration in solving the brachytherapy seed localization problem in actual clinical context. We show an implementation and detailed experimental analysis on ground truth phantom and initial results on patient data.

\section{METHOD}

Generally, intensity-based registration tends not to work between ultrasound and X-ray modalities, because in ultrasound anatomical structures are embedded in noisy and low contrast environment with little distinctive information about material density measured by X-ray. But implanting the prostate with seeds changes this situation advantageously. While TRUS images of an implanted prostate are hampered by noise and false positive and negative appearances, seeds carry enough distinctive information for intensity-based registration to hone in on the correct transformation. Still, the apparent straightforwardness of our method should not belie the investment of effort needed to make a workable clinical tool, despite the availability of technical components.

\section{Ground-truth Phantom:}

Figure 2 shows our experimental ground truth phantom setup. We assume that the process and notations are familiar from basic surgical navigation literature. We made a realistic implant in a phantom (CIRS, Norfolk, VA) with 48 seeds, arranged a set of $1 \mathrm{~mm}$ CT fiducials on the container box, and acquired a CT volume with $0.3 \mathrm{~mm}$ pixel size and $0.6 \mathrm{~mm}$ slice thickness. We carefully segmented the six fiducials attached to the walls of the phantom. The CT fiducials were also localized with a calibrated pointer (Traxtal Inc., Toronto, ON) and Certus optical tracker (NDI, Waterloo, ON) with respect to the DRB coordinate on the phantom. Using tracked TRUS acquisition, we scanned the phantom systematically with translational motion and continuous image capture. The in-plane pixel size was $0.14 \times 0.13 \mathrm{~mm}^{2}$. Inter-frame spacing was about $0.5 \mathrm{~mm}$. Following filtering, an 8-bit image volume was compounded from the 2D slices. Finally, we transformed the positions of seeds segmented in CT images to the TRUS coordinate system, thereby defining the ground truth (see Figure 2) for the registration. In order to maximize registration and tracking accuracy, the fiducials and tracking bodies were arranged so that their centroids fell close to the center of the prostate.
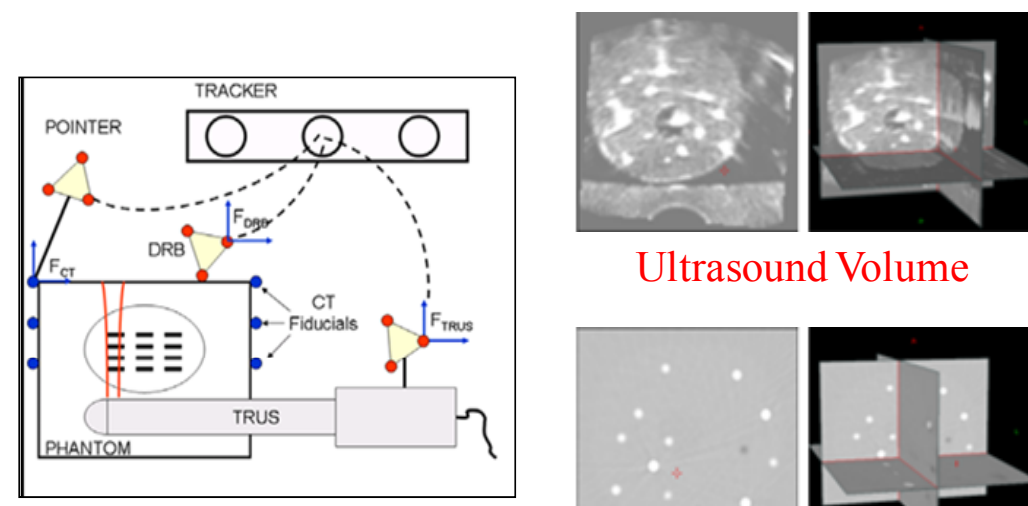

Ultrasound Volume

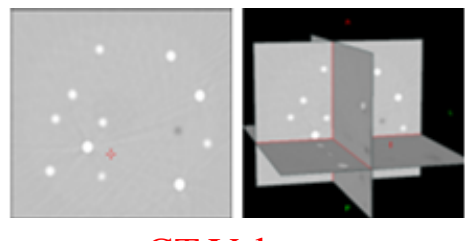

CT Volume

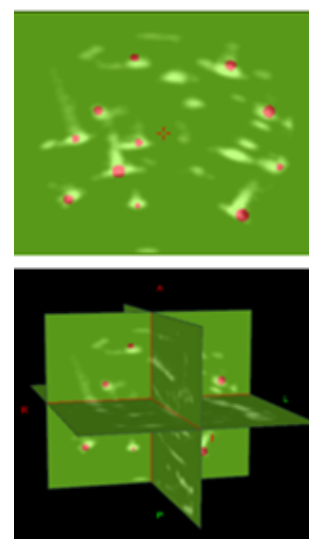

Ground Truth

Figure 2. Ground Truth Phantom. TRUS and CT images are shown in the $1^{\text {st }}$ and second images and the final ground truth overlay is shown in the last image.

Ultrasound Filtering: We tested and compared various filters on TRUS to suppress artifacts and enhance seed-like regions without explicit segmentation (see Figure 3). The baseline for comparison is no filtering (US-0). US-1 is a noise reduction filter based on two successive thresholdings followed by removal of regions whose area is smaller than that of a typical seed. The threshold values are obtained based on the average intensity of the pixels. US-2 is a phase congruency filter. Previously, Hacihaliloglu et al. used phase congruency (PCON) for detecting bone edges in 
ultrasound..$^{13}$ PCON evaluates features based on phase rather than amplitude information. Since it gives a measure of significance for each point invariant to image brightness or contrast, a constant and uniform threshold can be applied to extract feature points from the phase information. ${ }^{14}$ We calculate the PCON at each pixel of each image in order to measure phase symmetry. The more symmetrical the phase of a region is, the more likely it is to be a seed. The measure of symmetry is calculated as the weighted average resulting from even and odd symmetry filters. In this paper, we used the MATLAB implementation of PCON from Kovesi ${ }^{14}$. The beam profile filter (US-3) accounts for the finite thickness of the ultrasound beam and the focusing in the elevational and lateral directions, which make fidelity across the image nonuniform. ${ }^{15}$ In our experiment, the number of focal points in the lateral plane was set to two. We give more weight to the regions near the focal points and less to the ends of the image where the beam is less accurate. In US- 4 , we combine parallel noise reduction, phase congruency and beam profile filters in a Bayesian model, where each filter independently estimates "seedness" and their results are combined as in Scepanovic et al. ${ }^{16}$ In US-5 noise reduction is followed by phase congruency. In US-6, noise reduction is followed by beam profile filtering. Finally, in US-7 we cascade noise reduction, phase congruency, and beam profile filtering.
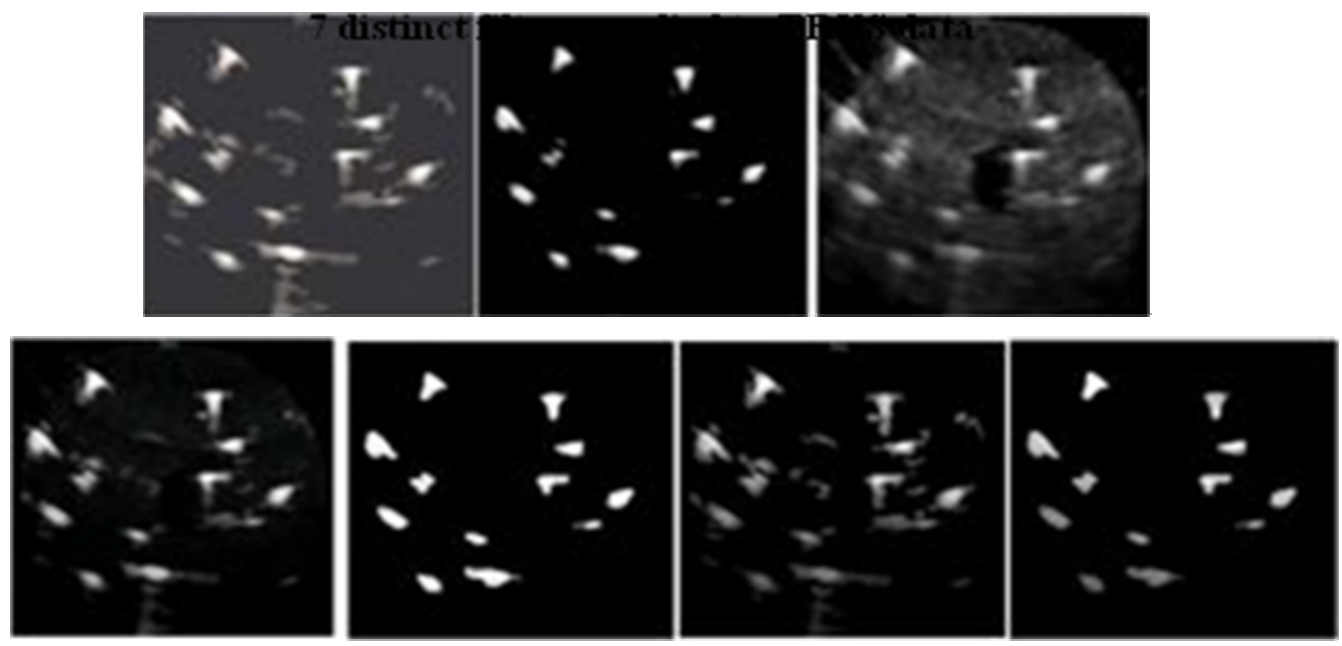

Figure 3. Seven distinct filters applied to TRUS images. (Top) (US1), phase congruency (US2) and beam width filter (US3). (Bottom row) A combination of filters was also applied: bayesian (US4), noise reduction with phase congruency (US5), noise reduction with beam width filter (US6) and lastly noise reduction with phase congruency and beam width filter (US7).

Registration Algorithm: An alternative to traditional volume-to-volume registration techniques is an algorithm that maps a $3 \mathrm{D}$ point cloud to a moving volume. Nevertheless, the goal of the registration method remains to determine the set of parameters of the transformation that optimizes a specified metric. For this particular technique, we assume a coordinate system of the fixed 3D point cloud (CT/Fluoroscopy) as a reference and search for a transform that will map points from the space of the fixed point set to the space of the moving image. The fixed point set coordinates are assigned intensity values as well and since we are considering using a binarized point cloud then we automatically assign an intensity value of 255 (i.e. sample coordinate (X,Y,Z,255). Two metrics were tested for this technique: the Normalized Correlation (NC) and Mean Squares metrics (MS) ${ }^{17}$. Clinically, we always have accurate and consistent initial guess for the registration. Standard patient positioning allows for estimating the main symmetry axes of the prostate, and alignment of the gravity centers of the TRUS volume and the seed cloud yields accurate guess for translation. ${ }^{18}$ One of the simplest and yet most powerful evolution strategies is the "one plus one evolution strategy", denoted by (1+1)-ES, and was used for algorithm optimization and parameter convergence.

\section{RESULTS}

Perturbations of magnitude $\pm 5 \mathrm{~mm}$ and $\pm 15^{\circ}$ were applied to the ground truth phantom for each of the 7 filters. A total of 25 perturbations for each filter were applied yielding a total of 200 simulations including the non-filtered ultrasound 
dataset. CT images were first thresholded in order to calculate center of seeds to produce a 3D point cloud. Here, we used MatLab to calculate the centroid. We then registered the perturbed point cloud to the TRUS images.

Phantom results: For the point-to-volume registration technique, results for the NC metric were less accurate when compared to the above volume-to-volume analysis. As seen in Table 1, filtering the TRUS data was beneficial, as 6 of the 7 filters yielded more precise TRE values than the unfiltered TRUS. In particular, US6 filter showed the best value of $0.54 \pm 1.36 \mathrm{~mm}$. The MS metric showed stronger statistical significance for average and standard deviation. Still, all filters yielded a final TRE below $1.0 \mathrm{~mm}$. In the false positive analyses the MS metric yielded excellent TRE below 2 $\mathrm{mm}$. The least accurate filter was achieved with the US3 (TRE of $0.87 \pm 0.11 \mathrm{~mm}$ ). With the NC metric, results deteriorated as the least precise value was again achieved with the US3 filter yielding (TRE of $1.97 \pm 1.34 \mathrm{~mm}$ ) for the false positive analysis. The un-optimized MATLAB code for P2V executed in 90 seconds, in average, on an Intel Core2, $2.4 \mathrm{GHz}$ dual-core computer.

Clinic Results: Clinical patient data was collected under ethics board approval. Here we present results on the first and so far our only patient dataset available. Seeds were reconstructed from four C-arm fluoroscopy images as a cloud of seeds using the method of Jain et al. ${ }^{18}$, compounded into a binary CT volume. We then performed P2V registration with both NC and MS metrics, with results shown in Figure 4 for the MS metric. A quick visual inspection reveals that there is no fluoroscopy seed without a white blotch in TRUS, suggesting that we do not have false negatives. As it was expected, there are some white blotches in TRUS without corresponding seed from fluoroscopy, suggesting the presence of multiple false positives. At the same time, the expert clinician had great difficulties in segmenting the dense series of TRUS slices with many seeds extending across consecutive images. For this particular patient, C-arm fluoroscopy reconstructed all the 81 implanted seeds. In the TRUS data, the expert clinician identified only 41 seeds with confidence, while 40 seeds could not be distinguished from noise and artifacts. Based on the 41 seeds positively segmented in TRUS, the average TRE was $2.86 \mathrm{~mm}$, with a standard deviation of $1.26 \mathrm{~mm}$.

Table 1. Mean and standard deviation of TRE in phantom trials for point-to-volume (P2V) registration (all units in mm)

\section{Target Registration Error (TRE)}

\begin{tabular}{c|cc}
\hline & NC & MS \\
Filter & $1.19 \pm 1.99$ & $0.40 \pm 0.17$ \\
US0 & $0.58 \pm 1.44$ & $0.70 \pm 0.22$ \\
US1 & $0.88 \pm 1.68$ & $0.49 \pm 0.12$ \\
US2 & $1.89 \pm 2.23$ & $0.56 \pm 0.12$ \\
US3 & $0.67 \pm 1.67$ & $0.38 \pm 0.19$ \\
US4 & $0.66 \pm 1.75$ & $0.65 \pm 0.24$ \\
US5 & $0.54 \pm 1.36$ & $0.88 \pm 0.17$ \\
US6 & $0.76 \pm 1.51$ & $0.83 \pm 0.21$ \\
US7 & &
\end{tabular}



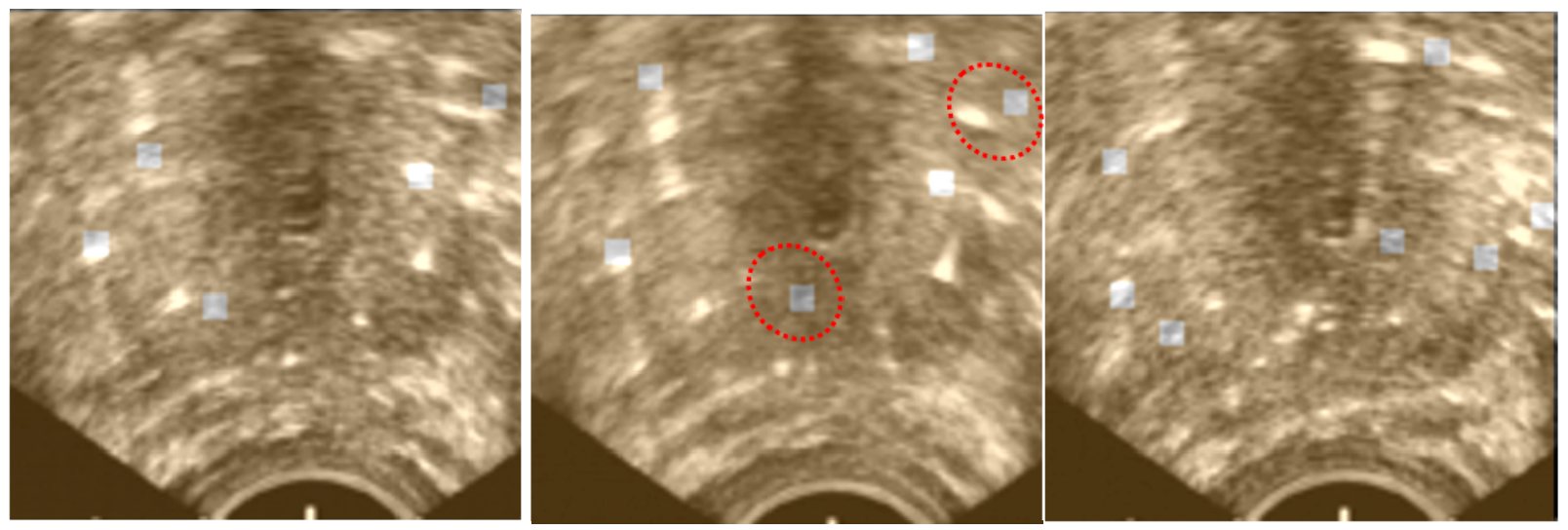

Figure 4. Clinical results of a registration of TRUS and seeds registered from multi-view fluoroscopy. All fluoroscopic seeds (marked with a square) except two align with white TRUS features within the $2 \mathrm{~mm}$ clinical limit. Two seeds in the center image (marked by the ovals) do not match with TRUS, possibly due to concurrent false positive and acoustic shadowing effects.

\section{DISCUSSION}

To our knowledge, there has been no study to quantify the effects of TRUS and fluoroscopy registration error on dosimetric quality, i.e. to analyze the effect of each translation and rotation on dose volume histograms. Until such results become available in the literature, we use the accuracy threshold applied for the seed localization error ${ }^{5,18}$, of which is about $2.0 \mathrm{~mm}$.

Intensity-based registration between TRUS and CT/fluoroscopy reconstructions of prostate implants was found to be excellent in phantom studies, in terms of TRE, capture range and robustness to false positive seed appearances in TRUS.

We favor NC for simplicity and speed. This clearly suggests that recovering the seed centroids is sufficient for registration and there is no need for a costly and complicated recovery of seed length and direction ${ }^{19}$, unless that is deemed useful for dosimetry purposes.

Surprisingly perhaps, filtering TRUS did not yield tangible advantages. According to Table 1, unfiltered TRUS (baseline US0) outperformed all but one filters. In any case, the differences were both statistically and clinically insignificant. Complicated and time-consuming filters, such as phase congruency (US2) and combination filters (US4-US7), seem especially unjustified. All filters, including the dummy US0, yielded a TRE below $1.0 \mathrm{~mm}$, (i.e. an accuracy that was close to the accuracy of the ground truth), which is almost negligible from a dosimetric perspective.

Our experience with early clinical data clearly and forcefully underlines the inherent difficulty of reliable validation based on explicit segmentation of seeds in TRUS. For many seeds, the expert clinician could not tell apart true seeds from noise in TRUS. After two weeks, the clinician repeated the task of seed identification in the same patient data and nearly half of all seed locations were picked differently, suggesting unreliable consistency in visual seed localization. A possible workaround might be applying multiple segmenters, but that is likely to fail as well. Earlier, Orio et al. reported the same difficulty ${ }^{20}$, as they were able to visually identify $20-25 \%$ of all the implanted seeds in TRUS. We established registration ground truth as suggested by Jain et al. in ${ }^{18}$, by pre-registration of a radiographic fiducial and the TRUS coordinate space. This approach, however, is not generally robust and, as Jain et al. mentioned, it may require compensation for biases emanating from multiple sources. 


\section{CONCLUSION}

A new point-to-volume intensity-based registration technique was developed to perform intraoperative registration between TRUS and 3D model of brachytherapy seeds. The system was fully evaluated on data collected from a prostate phantom implanted with 48 dummy seeds. For phantom analysis, TRE was consistently below clinical threshold $(<$ $2 \mathrm{~mm}$ ), capture range was significantly larger than the initial guess guaranteed by the clinical workflow, robustness was excellent to false positive seed appearances, and temporal performance was acceptable. Initial result on clinical data was adequate.

\section{ACKNOWLEGEMENTS}

We are grateful to Siddharth Vikal, MSc, for his valuable advice and assistance throughout this project and to Xiao Xiao $\mathrm{Ma}$, BSc, for medical illustration and Dr. David Gobbi for the ground truth phantom protocol and experimentation. Dr. Pascal Fallavollita was supported by a Postdoctoral Fellowship of the Ontario Ministry of Research. This research was also funded by the NSERC Idea to Innovation program and by Queen's University.

\section{REFERENCES}

[1] Jemal, A., Siegel R., Ward E., Hao Y., Xu J., Thun MJ., “Cancer statistics 2009," Cancer J Clin, 59(4), 225-49 (2009)

[2] Nag, S., Ciezki, J., Cormack, R., Doggett, S., DeWyngaert, K., Edmundson, G., et al., "Intraoperative planning and evaluation of permanent prostate brachytherapy: Report of the American Brachytherapy Society," International Journal of Radiation Oncology Biology Physics, 51, 1422-1430 (2001).

[3] Kumar, P., Good, R., Epstein, B., Hussain, M., and Bartone, F., "Fluoroscopy guided transperineal percutaneous permanent 125iodine implantation of prostate cancer," Radiat Med, 3(3), 161-167 (1985)

[4] Prestidge, B., Prete, J., Buchholz, T., Friedland, J., Stock, R., Grimm, P., and Bice, W., "A survey of current clinical practice of permanent prostate brachytherapy in the United States," International Journal of Radiation Oncology Biology Physics, 15, 461-465 (1998)

[5] Jain, A., Zhou, Y., Mustafa, T., Burdette, E. C., Chirikjian, G. S., Fitchinger, G., "Matching and reconstruction of brachytherapy seeds using the Hungarian algorithm (MARSHAL)," Medical Physics, 32(11), 3475-3492 (2005)

[6] Tutar, I., Narayanan, S., Lenz, H., Nurani, R., Orio, P., Cho, P., Wallner, K., and Kim, Y., "Seed-based ultrasound and Fluoroscopy registration using iterative optimal assignment for intraoperative prostate brachytherapy dosimetry," Proc. SPIE Medical Imaging, 6509, 650914 (2007)

[7] Letourneau, D., Wong, J. W., Oldham, M., et al., "Cone-beam-CT guided radiation therapy: technical implementation," Radiother Oncol, 75(3), 279-286 (2005)

[8] Kaplan, I. D., Meskell, P., Oldenburg, N. E., et al., "Real-time computed tomography dosimetry during ultrasoundguided brachytherapy for prostate cancer," Brachytherapy, 5(3), 147-51 (2006)

[9] Zhang, M., Zaider, M., Worman, M. F., and Cohen, G. N., "On the question of 3D seed reconstruction in prostate brachytherapy: the determination of x-ray source and film locations." Phys. Med. Biol., 49, 335-345 (2004)

[10] French, D., Morris, J., Keyes, M., and Salcudean, S. E., "Real-time dosimetry for prostate brachytherapy using TRUS and Fluoroscopy," Medical Image Computing and Computer-Assisted Intervention, 3217, 983-991 (2004)

[11] Su, Y., Davis, B. J., Furutani, K. M., Herman, M. G., and Robb, R. A., "Seed localization and TRUS-Fluoroscopy fusion for intraoperative prostate brachytherapy dosimetry," Computer Aided Surgery, 12(1), 25-34 (2007)

[12] Chen, T.K., Thurston AD, Ellis R.E., et al., "A real-time freehand ultrasound calibration system with automatic accuracy feedback and control,” Ultrasound in Medicine \& Biology, 35(1), 79-93 (2009) 
[13] Hacihalilogue, I., et al., "Enhancement of bone surface visualization from 3D ultrasound based on local phase information," IEEE Ultrasonics Symp, 21-24 (2006)

[14] Kovesi, P., "Image feature from phase congruency," Journal of Computer Vision Research, 1(3), 1-27 (1999)

[15] Chen, T.K., et al., "A real-time ultrasound calibration system with automatic accuracy control and incorporationof ultrasound section thickness," Proc. Of SPIE, 6918(1), 69182A (2008)

[16] Scepanovic, D., Kirshtein, J., Jain, A.K., Taylor, R.H., "Fast algorithm for probabilistic bone edge detection," SPIE Medical Imaging, 5747, 1753-1765 (2005)

[17] Yoo ,T.S., Ackerman M. J., Lorensen W. E., et al., "Engineering and Algorithm Design for an Image Processing API: A Technical Report on ITK - The Insight Toolkit," Proc. of Medicine Meets Virtual Reality, 85,586-592 (2002)

[18] Jain, A. K., Deguet, A., Iordachita, I., et al., "Intra-operative 3D guidance in prostate brachytherapy using an average c-arm," Medical Image Computing and Computer-Assisted Intervention, 4792, 9-17 (2007)

[19] Zhou, Y., Jain AK, Chirikjian GS, Fichtinger G., "Incorporating Seed Orientation in Brachytherapy Implant Reconstruction," Proc. SPIE Medical Imaging, 6141, 438-452 (2006)

[20] Orio, P.F., Tutar, I.B., Narayanan S., Arthurs S., Cho P.S., Kim, Y., Merrick, G., Wallner, K.E., "Intraoperative ultrasound-fluoroscopy fusion can enhance prostate brachytherapy quality," International Journal of Radiation Oncology Biology Physics, 69(1), 302-7 (2007)

[21] Karimaghaloo, Z., Abolmaesumi, P., Ahmidi N., Chen, T. K., Gobbi, D. G., Fichtinger G., "Intra-operative Localization of Brachytherapy Implants Using, Intensity-based Registration," Proc. SPIE Medical Imaging, 10 (2), 726139.1-726139.8 (2009) 